\title{
A baitbox for all seasons: temporal shifts in a vector's propagule supply characteristics and implications for invasion ecology
}

\author{
Amy E. Fowler ${ }^{1,2, *}$, April M. H. Blakeslee ${ }^{2,3}$, João Canning-Clode ${ }^{2,4}$, \\ Michele F. Repetto ${ }^{2,5}$, Gregory M. Ruiz ${ }^{2}$, A. Whitman Miller ${ }^{2}$ \\ ${ }^{1}$ Department of Environmental Science and Policy, George Mason University, Fairfax, VA 22030, USA \\ ${ }^{2}$ Marine Invasions Laboratory, Smithsonian Environmental Research Center, Edgewater, MD 21037, USA \\ ${ }^{3}$ Biology Department, East Carolina University, Greenville, NC 27858, USA \\ ${ }^{4}$ MARE - Marine and Environmental Sciences Centre, \\ Agência Regional para o Desenvolvimento da Investigação Tecnologia e Inovação (ARDITI), Madeira 9020-105, Portugal \\ ${ }^{5}$ Biology Department, Temple University, Philadelphia, PA 19122, USA
}

\begin{abstract}
Invasion dynamics are influenced by both vector operation and propagule pressure. Which propagules are entrained in a vector depends on how, where, and when a vector operates, but the timing and effects of vector operations on species delivery patterns is poorly resolved. Using the live marine baitworm trade, we tested vector selectivity across 3 boreal seasons (summer 2011, fall 2011, and spring 2012). We compared macroinvertebrate assemblages at the source (Maine, US east coast field) and in baitboxes upon delivery (Mid-Atlantic distributors, US east coast) and quantified live and dead biota to test for interactive effects of season and vector stage (i.e. source vs. destination) on per capita abundance, species richness, diversity, functional richness, and community composition. In all, we identified 46262 hitchhiking macro-organisms from 56 distinct taxa. Among live biota, taxonomic richness, functional group richness, and abundance differed by vector stage and season. Community composition showed seasonality for functional groups, but not for taxonomic groups. Vector stage affected dead community composition more than season, implying that vector operations (i.e. handling at source and during shipping) filter species transfers differentially. Dead communities were typically composed of the most abundant live organisms in the same baitboxes, emphasizing how important propagule pressure is to successful transport. Some combinations of 5 key functional traits (body size, feeding mode, growth form, modularity, and motility) were associated with increased survival during vector transfer. Successful species transfers are correlated with specific functional traits and propagule pressure, both of which are influenced by seasonal variation.
\end{abstract}

KEY WORDS: Invasive species $\cdot$ Functional diversity $\cdot$ Marine bait $\cdot$ Propagule pressure

\section{INTRODUCTION}

A central goal in ecology is understanding factors that shape the distribution of species, including those that enable species to successfully invade novel communities (Elton 1958). Prior to humanmediated species transfers over the last few centuries, dispersal occurred via natural mechanisms and was subject to a multitude of natural barriers

${ }^{*}$ Corresponding author: afowler6@gmu.edu and abiotic and biotic processes (Gaylord \& Gaines 2000, Forrest et al. 2009, Luiz et al. 2012). Human activities have thus superseded natural movement barriers for a significant number of organisms around the globe, a trend that has increased significantly in recent decades (e.g. Hughes et al. 2015, Ruiz et al. 2015, Seebens et al. 2016). This has created opportunities to test fundamental questions about what affects variation (selectivity) among

() The authors 2020. Open Access under Creative Commons by Attribution Licence. Use, distribution and reproduction are unrestricted. Authors and original publication must be credited. 
taxa, based on functional or physiological attributes, in successful invasions.

In human-mediated dispersals, the transfer mechanism (i.e. vector) is the first of 4 step-wise stages in classic invasion models, after which organisms that survive transport are introduced (stage 2), may establish (stage 3), and spread (stage 4) (Carlton \& Ruiz 2015). The scope and scale of these vectors are spatially and temporally variable in quantity of material transported, transit, and season (Carlton \& Ruiz 2015), and the vector itself can impose severe limits on the number and genetic composition of species introductions, where only the most tolerant taxa survive within-transit abiotic and biotic conditions (Miller \& Ruiz 2009). Furthermore, many vectors operate year-round, adding to the complexity surrounding the successful entrainment and transfer of taxa. In particular, temperate source regions experience marked seasonal changes in species abundance and diversity, which influence source pools of taxa that may become entrained in a vector (Stachowicz \& Tilman 2005, Stachowicz \& Byrnes 2006). For many vectors, the lack of robust data on vector selectivity for particular species or organismal traits can be attributed to these unknown or understudied source pools, limiting understanding of which factors affect the likelihood of invasions (Zenni \& Nuñez 2013).

While many biological invasion studies compare attributes of accidentally introduced successful and failed species within taxonomic groups (reviewed in Miller \& Ruiz 2009, Zenni \& Nuñez 2013), most do not explicitly consider the role of the vector as a selective agent in this process. The majority of what we know about vector selectivity for successful, accidental introductions comes from just a few studies with data on both source pools and recipient assemblages. For example, in aquatic systems, ballast water and hull fouling are efficient transport mechanisms, yet many entrained organisms experience high rates of natural mortality due to harsh conditions during transport (e.g. variable temperature, salinity, food availability) (Wonham et al. 2001, Verling et al. 2005, Hewitt et al. 2009, Lee \& Chown 2009). In general, species diversity and abundance declines as voyage time increases, but organismal assemblages may also exhibit seasonal differences in densities and compositions (Verling et al. 2005, Lee \& Chown 2009, Simkanin et al. 2009, Simard et al. 2011).

In one of the few non-ballast/hull fouling studies to date, Miller et al. (2007) investigated the intentional movement of oysters Crassostrea virginica from the east coast of North America (source region) to San Francisco Bay, CA, USA (recipient region). Among
93 molluscan species entrained in the vector, the 12 species that successfully invaded shared attributes of low-salinity tolerance, high population abundance, and direct development reproduction (Miller et al. 2007). Only one of these attributes, high population abundance, was likely directly linked to the vector in the form of propagule pressure, while others were organismal functional traits that contributed to colonization success.

In this study, we comprehensively tested the role of vector selectivity in determining the macroinvertebrate community composition and mortality during transit in a highly tractable system. Specifically, we evaluated and characterized seasonal changes in transfer and survivorship of a diverse macroinvertebrate community associated with marine bait. Our model vector, the Maine live bait trade, is a yearround, active vector using marine baitworms found in temperate regions of eastern North America that are packaged together with live algae and shipped to coastal locations in the continental USA and abroad (e.g. Italy, France, and Spain) (Creaser et al. 1983, Crawford 2001). What makes this vector unique is that it can be assessed at all stages of the pathway, providing a powerful way to test vector selectivity. Recent work has highlighted this vector as a potential pipeline of biological invasion for over 110 taxa of intertidal marine macro- and micro-organisms (Cohen 2012, Haska et al. 2012, Blakeslee et al. 2016, Fowler et al. 2016). Previous work has described changes in the abundance, taxonomic richness, and diversity of hitchhikers at 3 steps along the supply chain (source, baitboxes, and bait bags) during a single boreal season (summer) (Fowler et al. 2016). Here, we evaluate those same community-level changes at the source and recipient region across 3 seasons. In addition, we assess functional group composition (i.e. traits that influence habitat and resource use) in the transported community. Such functional understanding is necessary in the context of species interactions and the influence on overall ecosystem function in recipient regions (Downing \& Liebold 2002). Incorporating these metrics allow us to explore how seasonal niches and vector selectivity underlie overall patterns of propagule pressure in this potent vector.

\section{MATERIALS AND METHODS}

\subsection{Study system}

In this model system, marine polychaete worms (primarily bloodworms Glycera dibranchiata Ehlers 
1868) are packaged in 'wormweed' algae (Ascophyllum nodosum (Linnaeus) Le Jolis 1863 ecad scorpioides) after hand collection from intertidal mudflats along coastal Maine, USA. Wormweed is preferentially harvested to cushion and hydrate live baitworms. Packaged worms are then sent to locations nationally and internationally to Europe via overnight air delivery (Creaser et al. 1983, Crawford 2001, Sá et al. 2017). Wormweed naturally forms a complex, floating, 3-dimensional surface that provides habitat and resources and ameliorates abiotic stressors for macroinvertebrates (Dijkstra et al. 2012, Tyrrell et al. 2012). Because the baitworm trade is keenly focused on keeping worms alive, the survivorship of hitchhiking macroinvertebrates on the wormweed is also enhanced, thereby transporting relatively intact communities of all life stages, including gravid females (Fowler et al. 2016). Once purchased by anglers, bait and wormweed can be accidentally (or intentionally) released into the environment, providing opportunities for establishment and spread (Lau 1995).

\subsection{Sampling}

To assess the seasonal richness, abundance, and diversity of marine invertebrates transported through this vector, we quantified the community at 2 discrete stages, before and after transfer, where Maine coastal habitats are the primary source of worms and seaweed and the Mid-Atlantic US coast is the recipient region. The Mid-Atlantic region is composed of the states of Delaware, New Jersey, Pennsylvania, Maryland, Virginia, and North Carolina, where previous work (Fowler et al. 2016) examined the wormweed vector from source (Maine) to recipient bait shops in coastal regions of these states (excluding inland Pennsylvania) during the summer season. Here, we focused on one recipient region, Maryland, where we could perform a more in-depth multi-season exploration. During summer (July) 2011, fall (October) 2011, and spring (May) 2012, we examined associated organisms found in both natural wormweed source habitats in Maine (e.g. saltmarsh and shoreline habitats) and in baitboxes packed with wormweed which had been purchased directly from Maine distributors and shipped to Maryland using standard packing and shipping procedures (per Fowler et al. 2016).

The field sample collection of wormweed occurred at 5 field sites in the Boothbay, Maine region that are actively used for commercial wormweed purposes (P. Thayer, Maine Department of Marine Resources, pers. comm.). These field collections were intended to represent both the source ecosystem and the harvested hitchhiker assemblages before they undergo any additional selection during packaging and transport. Wormweed was collected from 6 randomly positioned quadrats $\left(0.5 \mathrm{~m}^{2}\right)$ along a $30 \mathrm{~m}$ transect running parallel to the water's edge; if a quadrat did not contain wormweed, it was haphazardly re-positioned to the nearest location with wormweed present. Field-collected wormweed was combined and randomly divided into 3 replicate 0.951 units per site.

To sample wormweed upon arrival at recipient regions, we purchased a baitbox of 250 bloodworms from 5 baitworm distributors in the Boothbay region (over the phone from Maryland) every week for $3 \mathrm{wk}$ during each season. While each distributor varied in their packing and shipping methodologies (i.e. where wormweed was collected, how wormweed was stored and sorted, etc.; A. Fowler pers. obs.), the majority of shipments spent $<24 \mathrm{~h}$ in transit. Therefore, these baitboxes represent the variety of possible conditions in which Mid-Atlantic purchasers could receive the product. Wormweed from baitboxes was first separated from bloodworms and then divided into three $0.95 \mathrm{l}$ containers to create 3 replicates and provide a measure of the same sampling unit (volume) of algae collected in the field.

Post-shipment and field wormweed were rinsed in artificial saltwater (30 ppt) to dislodge associated organisms. This rinse water was then sieved $(63 \mu \mathrm{m})$ to retrieve dislodged biota. Wormweed was then visually inspected for biota; organisms were systematically removed, assigned as alive or dead, and preserved in $95 \%$ ethanol for analysis. Preserved organisms were examined under a stereomicroscope (40×), identified to the lowest possible taxonomic level based on morphological characters (to species if possible), and counted to provide measures of richness and abundance. Organisms were also assigned to functional groups using the following 5 dimensions that describe important physical and life history characteristics (adapted from Wahl 2009, Canning-Clode et al. 2008): body size (small, medium, large, or very large); growth form (encrusting, massive, bushy, or filamentous); trophic type (autotroph, suspension feeder, deposit feeder, predator, or grazer); modularity (solitary or colonial); and motility (attached, crawling, swimming, drifting, or burrowing).

\subsection{Data analysis}

Statistical analyses were performed separately for live and dead organisms encountered at each vector 
stage. Analyses, using univariate and multivariate methods, were restricted to marine macroinvertebrates due to the dominance and relatively high taxonomic resolution for this group (Table S1 in the Supplement at www.int-res.com/articles/suppl/m641 p013_supp.pdf). One distributor used unprinted newspaper rather than wormweed for packaging; data from this distributor were extreme outliers due to lack of associated biota and were thus excluded from analyses.

To assess both taxa richness and evenness, the Shannon-Wiener (S-W) diversity index was calculated. The proportion of species (i) relative to the total number of species $\left(p_{i}\right)$ was multiplied by the natural logarithm of the proportion $\left(\ln p_{i}\right)$, summed across species, and multiplied by -1 . Functional groups of species were used to compare the functional evenness $\left(\mathrm{FE}_{\text {; }}\right.$ i.e. regularity with which the functional space is filled by species, weighted by abundance; Villéger et al. 2008) and functional dispersion (FD; i.e. weighted mean distance in multidimensional trait space of individual species to the weighted centroid of all species, where weights correspond to the relative abundances of the species; Laliberté \& Legendre 2010) of live macroinvertebrate communities across seasons and stages. FE decreases either when abundance is less evenly distributed among species or when functional distances among species are less regular (Villéger et al. 2008). Relatively high values of FD suggest that there is higher niche differentiation and lower resource competition, indicating increased ecosystem function (Mason et al. 2005). Functional indices were computed using the 'FD' package (Laliberté et al. 2014) in R v.3.5.0 (R Development Core Team 2018).

Generalized linear mixed models (GLMMs) tested for differences across vector stages and seasons for taxonomic (abundance, percent live, richness, and S$\mathrm{W}$ diversity) and functional group (richness, FE, and FD) classifications in live and dead communities. Vector stage (2 levels: Maine field source, Maryland post-shipment distributor), seasonality (3 levels: spring, summer, fall), and vector $\times$ season were fixed factors, along with the random effects of field site/ distributor within each vector stage. Data were fit with a negative binomial probability distribution and log-link function (live and dead abundance, dead functional group richness), Poisson distribution and log-link function (live and dead taxonomic richness), gamma probability distribution and log-link function (percent live, live S-W diversity, live functional group richness, live FE, and live FD), or normal distribution and identity-link function (dead S-W diversity). Mul- tiple comparisons were adjusted using a sequential Bonferroni and significance level of $\alpha=0.05$. All univariate analyses were conducted in SPSS Statistics v.24 (IBM Corporation).

To compare community assemblages of macroinvertebrates across vector stage and season, taxonomic and functional data were contrasted in multivariate analyses using PRIMER v.6 (Clarke \& Gorley 2006). Abundance was square-root transformed to decrease the influence of very abundant species, and transformed values were used to create a resemblance matrix using S17 Bray-Curtis similarity index (Bray \& Curtis 1957). Non-metric multidimensional scaling plots were generated to visualize differences among stages and seasons, and a 2 -factor permutational multivariate analysis of variance and multiple pairwise comparisons determined if significant differences in taxa assemblages existed. The square root of estimates of components of variation was used for comparing the amount of variation attributable to specific terms in the multivariate PERMANOVA models (Anderson et al. 2008). Similarity percentage analyses were conducted to determine which taxa were driving the differences observed. Samples lacking dead organisms were excluded from dead community analyses ( 2 summer and 4 fall field samples).

To minimize underestimation of live taxonomic richness, rarefaction analyses constructed accumulation curves and second-order Jackknife estimators (Jack2; Smith \& van Belle 1984) using EstimateS v.9.1.0 (Colwell 2013). Jack2 is the best estimator for characterizing marine benthic communities (Canning-Clode et al. 2008). Because a clearly asymptotic accumulation curve indicates complete capture of total taxa richness in a population (Gotelli \& Colwell 2001), Jack2 estimator curves and taxa accumulation curves converging on the same asymptote reflect adequate sampling (Walther \& Morand 1998).

\section{RESULTS}

\subsection{Analysis of living taxa}

In total, we identified 78 live taxa (62 in baitboxes; 65 in the field) and 42735 live macro-organisms; $97.8 \%$ of these were marine invertebrates, which constituted $96.9-99.2 \%$ of the diversity across each season (Table S1). Some live marine invertebrates ( $\mathrm{n}=20$ taxa) were only present in certain seasons, with 8 different taxa collected exclusively during each spring and summer and 4 taxa found only during fall (Table S1). Most taxa only present during cer- 
tain seasons were found in field samples only $(40 \%)$, followed by baitboxes only ( $35 \%)$, and both field and baitboxes $(25 \%)$.

\subsubsection{Mean abundance}

The mean number of live marine macro-invertebrates was significantly higher in field samples than baitboxes across all seasons (Fig. 1a). The abundance of live organisms found in field samples and baitboxes did not differ across seasons, and only vector stage significantly influenced mean abundance (GLMM, $\left.F_{1,75}=16.54, \mathrm{p}<0.001\right)$. The same suite of live organisms drove the high abundances in field samples for each season and included isopods (Jaera albifrons), marine mites (e.g. Halacaridae), flatworms, and free-living nematodes (Table S1). Some taxa were more abundant in the field and baitboxes during particular seasons (e.g. littorine snails in sum- mer, seed shrimp in spring, and gammarid amphipods in fall; for species names and more information see Table S1).

\subsubsection{Mean taxonomic richness and S-W diversity index}

The interactions of vector stage $\times$ season strongly influenced taxonomic richness (GLMM, $F_{2,75}=4.97$, $\mathrm{p}=0.009$; Fig. 1b). Mean taxonomic richness was not different between field and baitbox samples in summer, but significantly decreased between vector stages in spring and fall, with fall baitboxes having significantly lower richness compared to other seasons (Fig. 1b). Rarefaction analyses indicated that a much larger species pool existed in the field across all seasons than was included in our samples, with the difference between expected and observed species richness ranging from $26-49 \%$. Only samples from
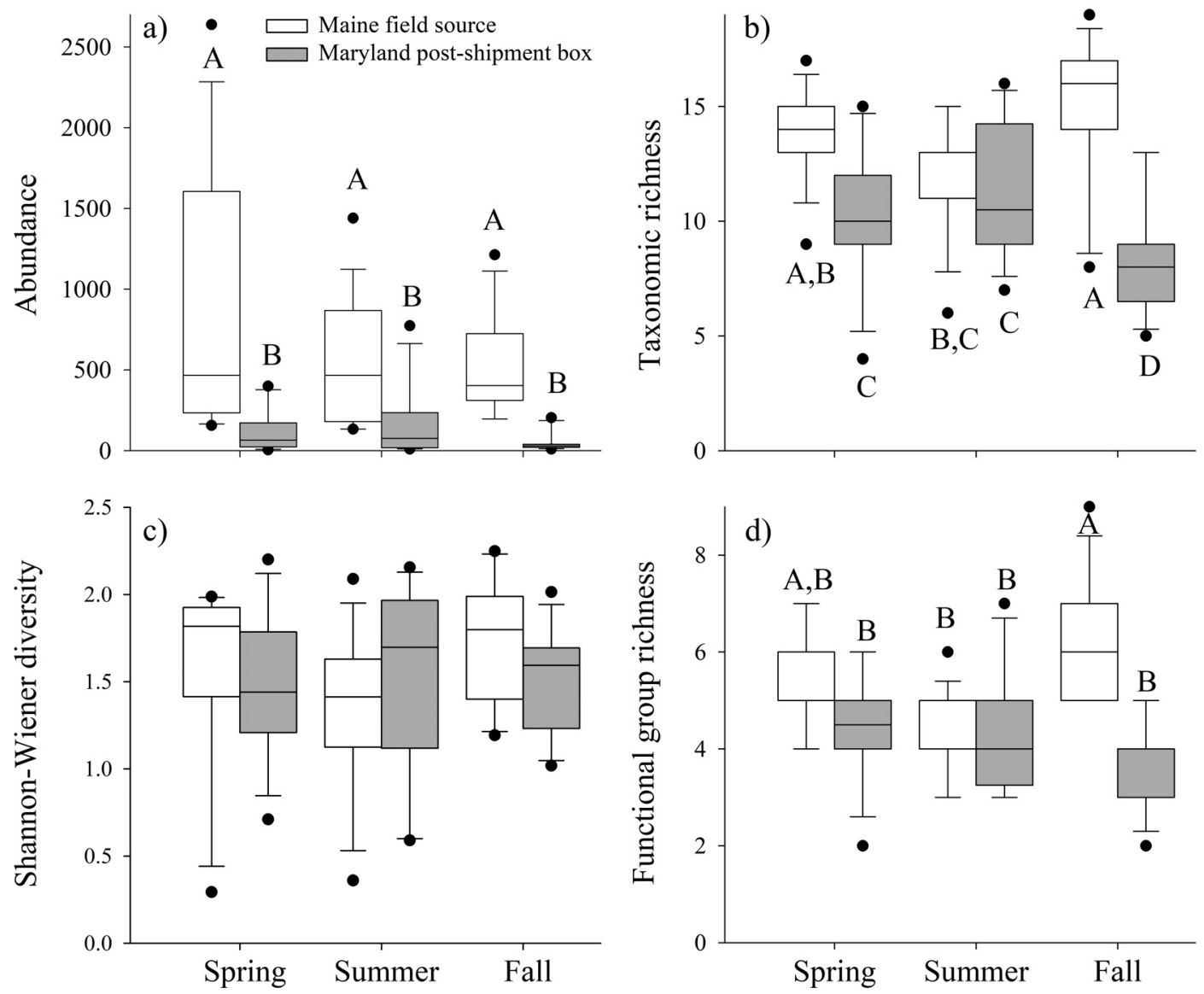

Fig. 1. Mean ( \pm SE) (a) abundance, (b) taxonomic richness, (c) Shannon-Wiener diversity, and (d) functional group richness of live marine macroinvertebrates found in replicate $0.95 \mathrm{l}$ containers during each season in Maine field source samples and Maryland post-shipment distributor baitboxes. Note different $y$-axes. Bars that share letters indicate lack of statistical difference at $\alpha=0.05$. Upper and lower limits of box plots: $75^{\text {th }}$ and $25^{\text {th }}$ percentiles; horizontal bars at the ends of the lines outside the boxes: $90^{\text {th }}$ and $10^{\text {th }}$ percentiles; horizontal line within the box: median; black circles: values outside the $90^{\text {th }}$ and $10^{\text {th }}$ percentile boundaries 
summer baitboxes captured the majority of taxa (expected and observed difference of $10 \%$; Table S2).

S-W diversity did not show significant patterns across vector stages or seasons (Fig. 1c). In fact, during summer, higher diversity was observed in baitboxes than field samples, contrary to trends observed during the other 2 seasons (Fig. 1c).

\subsubsection{Mean functional richness}

Seasonality in vector stages also strongly shaped patterns of functional group richness (GLMM, $F_{2,75}=$ $12.20, \mathrm{p}<0.0001)$. Similar to trends observed in taxonomic richness, mean functional group richness decreased from the field to baitboxes (Fig. 1d), however this was only significant during fall. Rarefaction analyses indicated that a much larger functional pool existed at both stages in summer and fall than was included in our samples (Table S2). Samples from baitboxes captured all functional groups only during spring.

\subsubsection{Mean functional evenness and dispersion}

FE was significant for the interaction of vector stage $\times$ season $\left(G L M M, F_{2,72}=3.76, p=0.028\right)$, driven by the lowest evenness during the summer field sampling which was composed mainly of oligochaetes (Fig. 2). Similarly, FD was defined by vector stage $\times$ season (GLMM, $\left.F_{2,75}=8.91, \mathrm{p}<0.0001\right)$. The relative abundances of the species were significantly lower during summer field and fall baitbox samples and significantly higher in spring and fall field samples (Fig. 2).

\subsubsection{Community composition}

Taxonomic community composition of live marine macro-invertebrates was strongly influenced by the interaction of vector stage and season (PERMANOVA, $F_{2,75}=2.4, \mathrm{p}=0.001$ ), with clear visual representation of the differences in taxa assemblages due to vector stage (Fig. 3a). Live assemblages in baitboxes were significantly different from one another across all seasons (PERMANOA pairwise tests, all $\mathrm{p}<0.01$ ), while only spring/summer and summer/ fall assemblages were significantly different from one another in field samples (PERMANOVA pairwise tests, all $\mathrm{p}<0.01$ ). The most taxonomically similar assemblages were collected in spring/fall, while field and baitboxes were the most dissimilar across all sea-

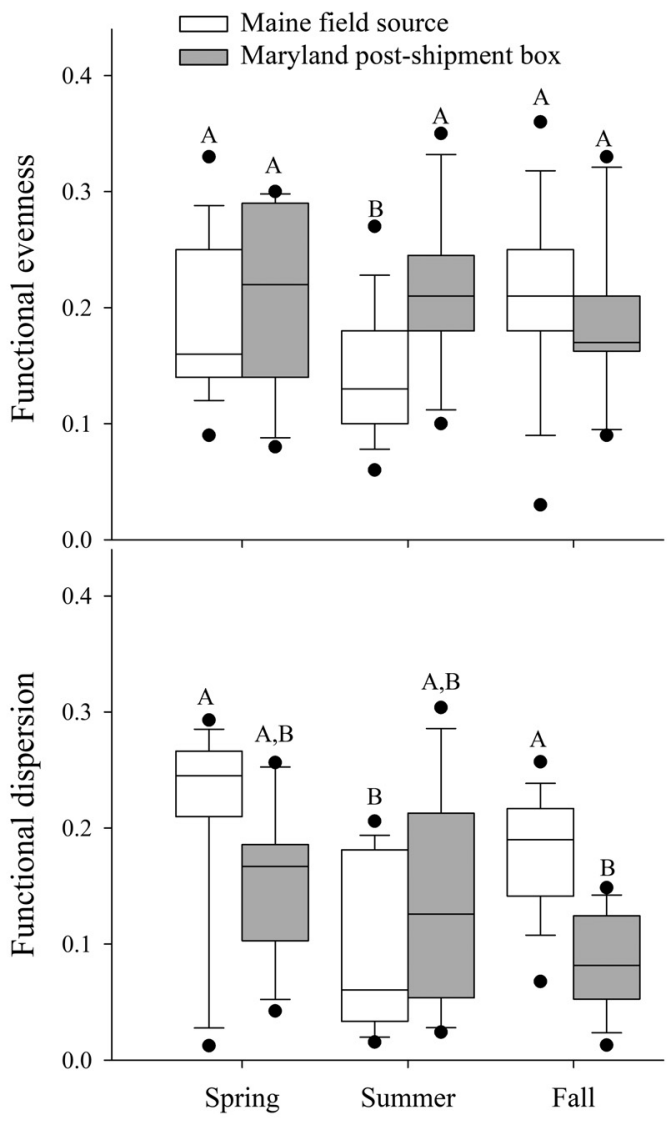

Fig. 2. Mean $( \pm \mathrm{SE})$ (a) functional evenness and (b) functional dispersion of live marine macroinvertebrates for all seasons in replicate 0.951 containers from Maine field source samples and Maryland post-shipment distributor baitboxes. Bars that share letters indicate lack of statistical difference at $\alpha=0.05$. Boxplot parameters as in Fig. 1

sons (Table S3). The top 4 taxa contributing to dissimilarity, Nematoda, Halacaridae, Littorina saxatilis (periwinkle snail), and $J$. albifrons, were responsible for $46.8-50.7 \%$ of dissimilarity between assemblages in pairwise comparisons. J. albifrons contributed the most $(14.4-16.8 \%)$ to average dissimilarity between stages, suggesting that fluctuations in $J$. albifrons contribute disproportionately to detected differences (Table S3).

Comparison of the square root of estimates of components of variation indicated that the vector $\times$ season effect was $30 \%$ stronger when considering functional rather than taxonomic community composition. Functional group assemblages were strongly influenced by the interaction of vector stage and season (PERMANOVA, $F_{2,75}=6.05, \mathrm{p}=0.001$ ), with clear visual representation of the differences in taxa assemblages due to vector stage and season (Fig. 3b). The most similar functional group compositions were collected 
Distributor, Spring * Field, Spring
Distributor, Summer * Field, Summer
Distributor, Fall * Field, Fall
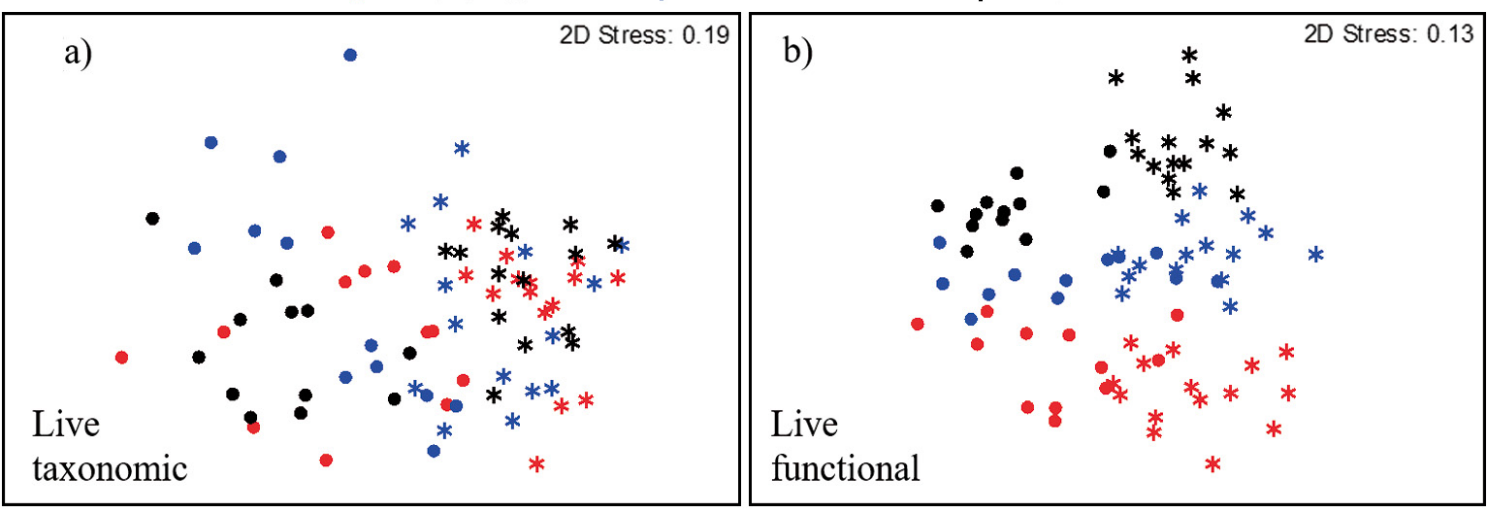

Fig. 3. Non-metric multidimensional scaling plots displaying taxonomic and functional similarity of live marine invertebrates sampled across seasons for Maine field source samples and Maryland post-shipment distributor baitboxes. PERMANOVA results for effect of vector $\times$ stage: (a) live taxonomic $F_{2,75}=2.4, \mathrm{p}=0.001$; (b) live functional $F_{2,75}=6.05, \mathrm{p}=0.001$

in summer/fall, while the most dissimilar were between assemblages observed in spring/fall (Table S3). Five functional groups were responsible for 77.3$86.6 \%$ of the dissimilarity between assemblages in pairwise comparisons. The functional group of medium body size, massive growth form, grazing trophic type, solitary modularity, and crawling motility (MMGSC) (e.g. amphipods, isopods, and some gastropods) contributed the most to average dissimilarity

Live in Maine field source

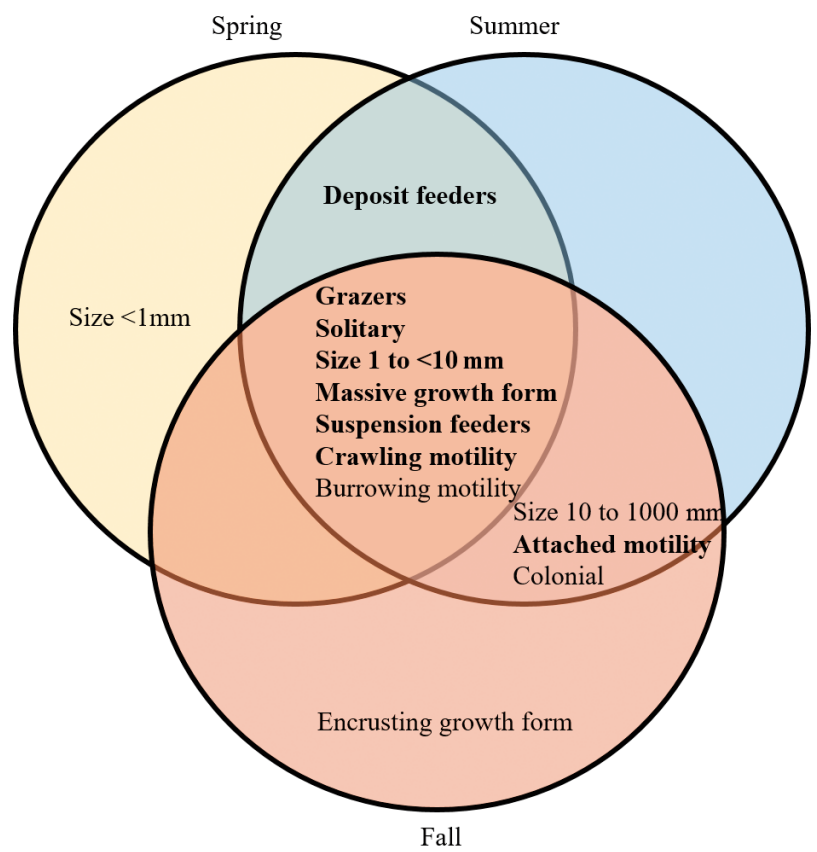

(18.8-30.9\%) in all cases except between assemblages observed in spring/fall (Table S3), suggesting that MMGSC drives the observed dissimilarity.

Of the functional traits observed for live macroorganisms, $52 \%(\mathrm{n}=9)$ were exclusive to a particular vector and season. Only $35 \%(n=6)$ were shared between all seasons and both vector stages (Fig. 4), including traits for body size (i.e. 1 to $<10 \mathrm{~mm}$ ), growth form (i.e. massive), trophic type (i.e. grazers

Arriving live in Maryland post-shipment bait boxes

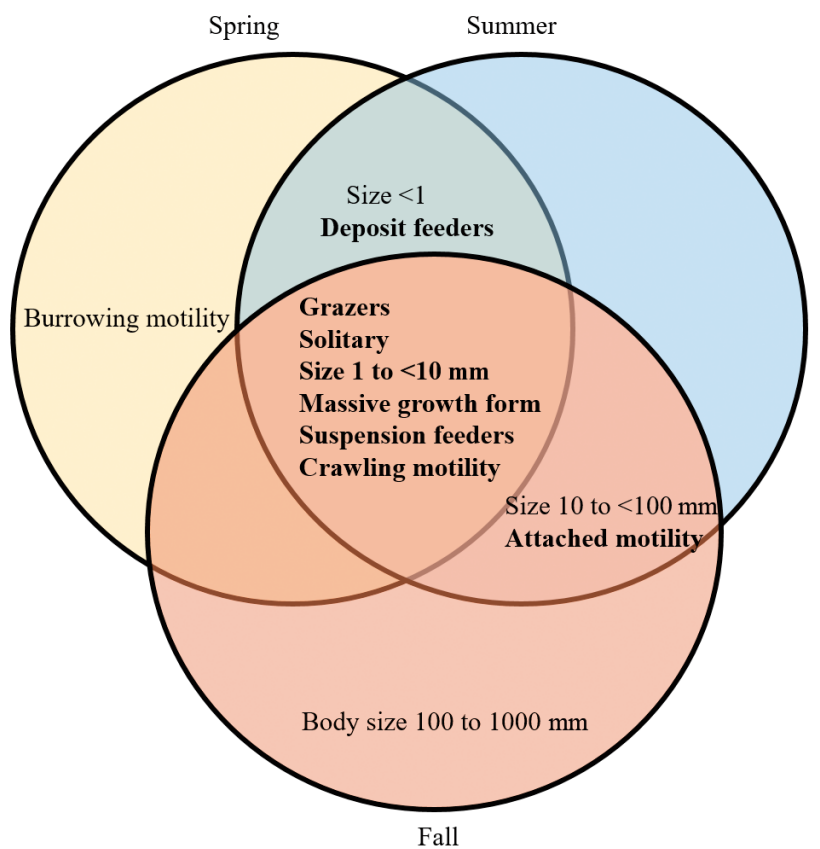

Fig. 4. Comparison of exclusive functional traits of live marine macro-organisms found in the source (Maine field) and recipient (Maryland post-shipment distributor baitboxes) regions during summer 2011, fall 2011, and spring 2012. Bold text: functional traits shared between the 2 vector stages. There are no unique functional traits at the intersection of fall and spring at either the source or recipient region 
and suspension feeders), modularity (i.e. solitary), and motility (i.e. crawling), all of which are traits of organisms such as isopods, amphipods, and gastropods (Fig. 4). The majority ( $56 \% ; n=5$ ) of non-shared traits centered around organismal size and exhibited a seasonal increase (i.e. $<1 \mathrm{~mm}$ in spring/summer, 10 to $<100 \mathrm{~mm}$ in summer/fall, and 100 to $1000 \mathrm{~mm}$ in fall) (Fig. 4). The other traits concerned motility (i.e. burrowing was observed in all seasons in the field but was exclusive to spring in baitboxes), modularity (i.e. colonial organisms were indicative of field summer/fall samples, but were not an important trait found in any seasons for baitboxes), and growth form (i.e. encrusting organisms were a key trait group in fall field samples, but were not key for any of the seasons for baitboxes) (Fig. 4).

\subsection{Analysis of dead taxa}

We identified 36 dead marine invertebrate taxa across seasons (29 in baitboxes; 22 in field collections) (Table S4). The majority (67\%) of field samples contained no dead taxa. Eight taxa were only found dead in baitboxes; some taxa $(n=14)$ were only present in certain seasons, with the highest numbers of dead taxa occurring in fall baitboxes (Table S1). Vector stage $\times$ season strongly influenced the abundance (GLMM, $\left.F_{2,75}=4.56, \mathrm{p}=0.013\right)$, taxonomic richness $\left(\right.$ GLMM $\left., F_{2,75}=3.63, \mathrm{p}=0.031\right), \mathrm{S}-\mathrm{W}$ diversity $\left(\right.$ GLMM $\left., F_{2,75}=5.68, \mathrm{p}=0.005\right)$, and community assemblage (GLMM, $F_{2,74}=1.57, \mathrm{p}=0.05$ ) of dead organisms (Fig. S1). The effect size of the taxonomic dead community was $16.7 \%$ smaller than that of the live community. Dead assemblages in baitboxes were significantly different from one another across all seasons, while only spring/summer and summer/fall assemblages were significantly different from one another in field samples (Fig. S2, Table S5). Four taxa (J. albifrons, the amphipod Apohyale prevostii, unidentifiable gammarid amphipods, and Capitellidae polychaetes) were responsible for $52.2-58.6 \%$ of the dissimilarity between assemblages (Table S5). J. albifrons contributed the most (29-39.7\%) to the dissimilarity, suggesting that fluctuations in dead J. albifrons may be responsible for detected differences.

Functional group richness was significantly different between vector stages (GLMM, $F_{1,75}=10.1, \mathrm{p}=$ 0.002), with higher dead richness in baitboxes across all seasons (Fig. S1). There were no dead individuals of LMPSC, LMSCA, XESCA, XMSCA, XBSCA, and XMSSA even though live specimens of each were found. (Letters refer to functional groups in the 5 di- mensions; see Section 2.2 and Table S1 in Fowler et al. 2016.) Two groups (XMSCA and XBSCA) were found alive in only one field sample, but others (LMSCA, XESCA, and XMSSA) were alive in multiple samples from baitboxes and the field and, in some cases (e.g. XMSSA), in large numbers. Although the effect size was $20 \%$ smaller than live communities, functional group community assemblage of dead taxa was also strongly influenced by the interaction of vector stage and season (PERMANOVA, $F_{2,74}=2.42, \mathrm{p}=$ 0.018; Fig. S2). Within baitboxes, spring/summer communities were not significantly different from one another (PERMANOVA pairwise tests, $t=0.465$, unique perms $=998, \mathrm{p}=0.902$ ); in field samples, spring/fall comparisons were not significantly different (PERMANOVA pairwise tests, $t=1.24$, unique perms $=996, \mathrm{p}=0.188$ ). The most similar functional group compositions were between assemblages collected in spring/summer, while the most dissimilar were between summer/fall. MMGSC contributed the most to the total average dissimilarity between stages (53.1-63.9\%) (Table S5).

\section{DISCUSSION}

Our study highlights the importance of considering seasonality and species' traits when modeling and interpreting invasion risk scenarios. The community of macroinvertebrates associated with the Maine live bait vector transitions seasonally in abundance, richness, and diversity (taxonomic and functional) in field source regions and recipient baitboxes, possibly stemming from population-level demography at the source (i.e. the highest propagule pressure from the field source pools in this vector is during spring and summer). Overall, both vector stage and season significantly influenced taxonomic and functional group richness, while abundance and survivability (i.e. \% live organisms) was driven by vector stage alone. Strikingly, there was no observable difference in community composition across seasons when using only taxonomic distinction, but seasonal differences arose when functional groups were considered, both in the field and vector. Therefore, for temperate vectors, seasonality can be a particularly important factor when combined with inherently high propagule pressure, strongly influencing the cumulative richness, diversity, and functional traits and impacting the relative outcomes of invasion opportunities and likelihood of invasion success.

The majority of previous studies examining the success and failure of introductions seasonally re- 
stricted analyses to single taxonomic groups in either the source and/or recipient region (e.g. Levine 2000, Romanuk et al. 2009, Chang et al. 2011). The Maine baitworm trade vector has the unique capacity to provide insights into unsuccessful introductions by quantifying presence/absence and mortality that occurs during transport (Fowler et al. 2016); in this case, unsuccessful taxa and functional groups are both those frequently found dead on arrival in baitboxes and those that were found in the field but not in baitboxes. Such results suggest that some taxa may be more susceptible to possible physiological stresses during transit inside the vector (i.e. increased mortality in the baitbox) and that others may have a lower probability of being entrained in the vector from the field.

In all cases, taxa with high death rates in the baitboxes were also among the most abundant live organisms in the same baitboxes (e.g. the isopod Jaera marina and functional group MMGSC), emphasizing the importance of propagule pressure in overall successful transport (Memmott et al. 2005, Miller et al. 2007, Blackburn et al. 2015). Put another way, despite high in-transit mortality, taxa that are entrained in large numbers may survive extensive mortality, resulting in substantial inoculations in recipient regions. Alternatively, some taxa do comparatively well during transit and have low percentages $(<10 \%)$ of dead individuals upon reaching the recipient region (e.g. littorine snails, marine mites, polychaete worms, nematodes, flatworms, and amphipods). In many cases, these taxa likely have higher inherent tolerances to environmental stressors (e.g. desiccation and temperature) that could enable them to withstand adverse conditions while in the vector. In natural ecosystems, abiotic tolerances often limit the distribution of organisms (Kennish 1990), so broad tolerance ranges of successful species seen alive in baitboxes are not surprising given the natural range of daily and seasonal stressors they endure in their native ecoregion (intertidal coast of Maine).

Functional traits, such as body size and trophic type, can influence organismal survival within the vector and may help shape patterns of invasion success and failure after transit (Romanuk et al. 2009, Schröder et al. 2009). Seasonal functional group differences were driven by shifts from the feeding mode of grazing and crawling motility in the spring and summer (i.e. gastropods and isopods) to deposit feeding and burrowing behaviors in the fall (i.e. polychaetes and nematodes) (Fig. 4), influenced by high abundances in the field. This can be seen in the significantly lower FE of the summer field sampling compared to all others (Fig. 2). Within baitboxes only, seasonal differences in functional groups were driven by organisms with a massive growth form, grazer feeding type, solitary modularity, and crawling motility (mostly isopods, amphipods, and gastropods; Fig. 4). FE of live organisms in baitboxes did not fluctuate among seasons (Fig. 2), indicating that available niche space was consistently filled and broadly distributed among functional groups (Mason et al. 2005). Overall, the lack of dominant functional groups showing clear seasonal patterns between live and dead assemblages indicates the efficiency of this vector and highlights a lack of severe mortality often seen with other vectors.

FD of live organisms should decrease from field to baitboxes due to the increasing influence of environmental and anthropogenic filtering (e.g. Algar et al. 2011, Lessard et al. 2016). FD and richness were significantly higher in the field than baitbox during spring and fall, demonstrating that after collection, handling, and shipping, baitbox assemblages comprised similar abundances of functionally similar species (i.e. trait convergence). However, field assemblages had higher variation in species abundance and functional traits, suggesting trait divergence (Valdivia et al. 2017). Higher FD in the field could suggest that (1) small-scale spatial differences in macroinvertebrate communities exist, highlighting the importance of the source pool location, and/or (2) the distributors themselves effectively act to 'homogenize' local field differences by combining wormweed from different sources. Unlike spring and fall, summer field samples had lower FD and richness than summer baitbox samples. One plausible explanation could be similarly high recruitment rates of dominant macroinvertebrates across the sampled field locations during the summer, which contribute to greater trait overlap among sites and thus lower FD in the summer samples than those observed during spring and fall (i.e. off peak recruitment).

The occurrence and relative distribution of novel functional traits in an ecosystem can vary across seasons, increasing the probability of establishment especially when abiotic conditions closely match source regions (Beche et al. 2006, Burkepile \& Hay 2008, Jousset et al. 2011). The observed seasonality in the community composition of functional groups may be directly related to the interplay of water temperature, natural reproduction, and larval recruitment dynamics at the source region. In turn, vectors operating at times that correspond with peak recruitment or reproduction at the source region likely transfer a higher abundance and diversity of propag- 
ules. This is especially true for taxa in functional group MMGSC (e.g. isopods and snails) that reproduce by direct development or brooding (Jones \& Naylor 1971). Brooding enables young to take advantage of good habitats already vetted by adults, but also can lead to high densities of recruits locally. This reproductive method is thought to have contributed to the invasion success of Littorina saxatilis (Carlton \& Cohen 1998, Chang et al. 2011, Panova et al. 2011) and mollusks in general (Miller et al. 2007).

After 70 yr of operation, the Maine live marine baitworm trade continues to successfully transfer live hitchhikers across continents and oceans. While we are beginning to gain an understanding of the potency of the vector, there are still many unanswered questions regarding the ecological impact of the vector and options to mitigate and/or stop the transfer of hitchhikers in algal packing materials. Invasions of the packing alga Ascophyllum nodosum (Miller et al. 2004), periwinkle snail L. saxatilis (Carlton \& Cohen 1998), and European green crab Carcinus maenas (Lau 1995, Cohen et al. 1995, 2001) are thought to continue to occur in the US west coast (and other regions) via this vector (Fowler et al. 2016). In fact, past work found these latter 2 globally invasive species to survive transport in baitboxes shipped to California (Blakeslee et al. 2016). This study provides the first overview of seasonal changes in the abundance and diversity of hitchhiking species, suggesting that seasonal windows of opportunity exist for the entrainment, transfer, and inoculation of species and have the potential to affect invasion success. However, further research on angler movement and release behavior is needed to completely understand additional sources of selection. In all likelihood, the differential opportunity for invasion via this vector, or others, may change inter-annually and even over decades. Under a changing climate, and as water temperatures continue to warm, seasonal differences in invasion opportunities may increase as the pool of available species increases due to corresponding changes in reproductive schedules, population demography, and, ultimately, propagule pressure. Similar vectors exist in other terrestrial and aquatic systems (e.g. the horticultural industry, ornamental plant trade, aquaculture of fish and oysters, and 'live rock' sold as part of the aquarium trade), where the trade item can host a variety of hitchhikers, including known noxious invaders (Grosholz et al. 2015, Ng et al. 2016, Patoka et al. 2016, Branford \& Duggan 2017); however, the scale at which the Maine live marine baitworm trade operates and the lack of information highlight the uncalculated risk of further successful introductions.
Vectors with repeated inoculations across seasons and years inherently increase propagule pressure and the probability of establishment (Drake \& Lodge 2004, Von Holle \& Simberloff 2005). Propagule pressure is an important ecological force, as even a series of failed invasions can result in reduced environmental resistance to the next invasion (Gonzalez et al. 2008). All of these factors combine to increase the probability of successful introduction of transported species and possible establishment in novel regions.

Acknowledgements. We thank the Smithsonian Environmental Research Center Marine Invasions Laboratory for their work on this project, P. Thayer for field and industry knowledge, N. Woodley for identifying insects, and J. T. Carlton for identifying 'unknowns'. We appreciate laboratory space, accommodations and support provided by the Darling Marine Center. This research was supported by Maryland Sea Grant awards NA10OAR4170072 and SA75 28120. Wormweed and associated organisms were collected under Maine Department of Marine Resources Special Licenses ME 2011-77-00 and ME 2012-14-01. J.C.C. was supported by national funds through FCT Fundação para a Ciência e a Tecnologia, I.P., under the Scientific Employment Stimulus - Institutional Call [CEECINST/00098/2018], and by FCT strategic project UIDB/04292/2020 granted to MARE.

\section{LITERATURE CITED}

Algar AC, Kerr JT, Currie DJ (2011) Quantifying the importance of regional and local filters for community trait structure in tropical and temperate zones. Ecology 92:903-914

Anderson M, Gorley RN, Clarke K, Anderson MJ and others (2008) PERMANOVA+ for PRIMER. Guide to software and statistical methods. PRIMER-E, Plymouth

*Beche LA, Mcelravy EP, Resh VH (2006) Long-term seasonal variation in the biological traits of benthic-macroinvertebrates in two Mediterranean-climate streams in California, USA. Freshw Biol 51:56-75

* Blackburn TM, Lockwood JL, Cassey P (2015) The influence of numbers on invasion success. Mol Ecol 24:1942-1953

Blakeslee AM, Fowler AE, Couture JL, Grosholz ED, Ruiz GM, Miller AW (2016) Vector management reduces marine organisms transferred with live saltwater bait. Manage Biol Invasions 7:389-398

* Branford SN, Duggan IC (2017) Grass carp (Ctenopharyngodon idella) translocations, including hitchhiker introductions, alter zooplankton communities in receiving ponds. Mar Freshw Res 68:2216-2227

Bray JR, Curtis JT (1957) An ordination of the upland forest communities of southern Wisconsin. Ecol Monogr 27: 325-349

*Burkepile DE, Hay ME (2008) Herbivore species richness and feeding complementarity affect community structure and function on a coral reef. Proc Natl Acad Sci USA 105: 16201-16206

Canning-Clode J, Valdivia N, Molis M, Thomason JC, Wahl M (2008) Estimation of regional richness in marine benthic communities: quantifying the error. Limnol Oceanogr Methods 6:580-590 
Carlton JT, Cohen AN (1998) Periwinkle's progress: the Atlantic snail Littorina saxatilis (Mollusca: Gastropoda) establishes a colony on a Pacific shore. Veliger 41: 333-338

Carlton JT, Ruiz GM (2015) Anthropogenic vectors of marine and estuarine invasions: an overview framework. In: Canning-Clode (ed) Biological invasions in changing ecosystems: vectors, ecological impacts, management and predictions. De Gruyter Open, Warsaw/Berlin, p 24-36

Chang AL, Blakeslee AM, Miller AW, Ruiz GM (2011) Establishment failure in biological invasions: a case history of Littorina littorea in California, USA. PLOS ONE 6:e16035

Clarke KR, Gorley RN (2006) PRIMER v6: user manual/tutorial. PRIMER-E, Plymouth

Cohen AN (2012) Aquatic invasive species vector risk assessments: live saltwater bait and the introduction of nonnative species into California. California Ocean Science Trust, Oakland, CA

Cohen AN, Carlton JT, Fountain MC (1995) Introduction, dispersal and potential impacts of the green crab Carcinus maenas in San Francisco Bay, California. Mar Biol 122: 225-237

Cohen AN, Weinstein A, Emmett MA, Lau W, Carlton JT (2001) Investigations into the introduction of nonindigenous marine organisms via the cross-continental trade in marine baitworms. A report for the US fish and wildlife service. San Francisco Bay Program, Sacramento CA. San Francisco Estuary Institute, Richmond CA

Colwell RK (2013) EstimateS: Statistical estimation of species richness and shared species from samples. Version 9 and earlier. User's guide and application. http://purl.oclc. org/estimates

Crawford SE (2001) Live rockweed (Ascophyllum) used as a shipping medium for the live transport of marine baitworms from Maine. In: Paust BC, Rice AA (eds) Marketing and shipping live aquatic products: proceedings of the second international conference and exhibition. University of Alaska Sea Grant, AK-SG-01-03, Fairbanks, AK, p 95-97

Creaser EP Jr, Clifford DA, Hogan MJ, Simpson DB (1983) A commercial sampling program for sandworms, Nereis virens Sars, and bloodworms, Glycera dibranciata Ehlers, harvested along the Maine coast. NOAA Tech Rep NMFS SSRF-767

Dijkstra JA, Boudreau J, Dionne M (2012) Species-specific mediation of temperature and community interactions by multiple foundation species. Oikos 121:646-654

Downing AL, Liebold MA (2002) Ecosystem consequences of species richness and composition in pond food webs. Nature 416:837-841

Drake JM, Lodge DM (2004) Global hot spots of biological invasions: evaluating options for ballast-water management. Proc R Soc B 271:575-580

Elton CS (1958) The ecology of invasions by plants and animals. Methuen Publishing, London

Forrest BM, Gardner JP, Taylor MD (2009) Internal borders for managing invasive marine species. J Appl Ecol 46: 46-54

Fowler AE, Blakeslee AM, Canning-Clode J, Repetto MF and others (2016) Opening Pandora's baitbox: a potent vector for biological invasions of live marine species. Divers Distrib 22:30-42

Gaylord B, Gaines SD (2000) Temperature or transport? Range limits in marine species mediated solely by flow. Am Nat 155:769-789
Gonzalez A, Lambert A, Ricciardi A (2008) When does ecosystem engineering cause invasion and species replacement? Oikos 117:1247-1257

* Gotelli NJ, Colwell RK (2001) Quantifying biodiversity: procedures and pitfalls in the measurement and comparison of species richness. Ecol Lett 4:379-391

*Grosholz ED, Crafton RE, Fontana RE, Pasari JR, Williams SL, Zabin CJ (2015) Aquaculture as a vector for marine invasions in California. Biol Invasions 17:1471-1484

*Haska CL, Yarish C, Kraemer G, Blaschik N, Whitlatch R, Zhang H, Lin S (2012) Baitworm packaging as a potential vector of invasive species. Biol Invasions 14:481-493

Hewitt CL, Gollasch S, Minchin D (2009) The vessel as a vector-biofouling, ballast water and sediments. In: Rilov G, Crooks JA (eds) Biological invasions in marine ecosystems. Ecological Studies No. 204. Springer-Verlag, Berlin, p 117-131

*Hughes KA, Pertierra LR, Molina-Montenegro MA, Convey $P$ (2015) Biological invasions in terrestrial Antarctica: What is the current status and can we respond? Biodivers Conserv 24:1031-1055

Kones MB, Naylor E (1971) Breeding and bionomics of the British members of the Jaera albifrons group of species (Isopoda: Asellota). J Zool 165:183-199

* Jousset A, Schmid B, Scheu S, Eisenhauer N (2011) Genotypic richness and dissimilarity opposingly affect ecosystem functioning. Ecol Lett 14:537-545

Kennish MJ (1990) Ecology of estuaries, Vol II: Biological aspects. CRC Press, Boca Raton, FL

* Laliberté E, Legendre P (2010) A distance-based framework for measuring functional diversity from multiple traits. Ecology 91:299-305

Laliberté E, Legendre P, Shipley B (2014) FD: measuring functional diversity from multiple traits, and other tools for functional ecology. R package version 1.0-12

Lau W (1995) Importation of baitworms and shipping seaweed: Vectors for introduced species? In: Sloan D, Christensen M, Kelso D (eds) Environmental issues: from a local to a global perspective. University of California Press, Berkeley, CA, p 21-38

Lee JE, Chown SL (2009) Temporal development of hullfouling assemblages associated with an Antarctic supply vessel. Mar Ecol Prog Ser 386:97-105

Lessard JP, Weinstein BG, Borregaard MK, Marske KA and others (2016) Process-based species pools reveal the hidden signature of biotic interactions amid the influence of temperature filtering. Am Nat 187:75-88

Levine JM (2000) Species diversity and biological invasions: relating local process to community pattern. Science 288 : 852-854

KLuiz OJ, Madin JS, Robertson DR, Rocha LA, Wirtz P, Floeter SR (2012) Ecological traits influencing range expansion across large oceanic dispersal barriers: insights from tropical Atlantic reef fishes. Proc R Soc B 279:1033-1040

Kason NW, Mouillot D, Lee WG, Wilson JB (2005) Functional richness, functional evenness and functional divergence: the primary components of functional diversity. Oikos 111:112-118

* Memmott J, Craze PG, Harman HM, Syrett P, Fowler SV (2005) The effect of propagule size on the invasion of an alien insect. J Anim Ecol 74:50-62

Miller AW, Ruiz GM (2009) Differentiating successful and failed invaders: species pools and the importance of defining vector, source and recipient regions. In: Rilov $G_{\text {, }}$ 
Crooks JA (eds) Biological invasions in marine ecosystems. Ecological Studies No. 204. Springer-Verlag, Berlin, p 153-170

Miller AW, Chang AL, Cosentino-Manning N, Ruiz GM (2004) A new record and eradication of the northern Atlantic alga Ascophyllum nodosum (Phaeophyceae) from San Francisco Bay, California, USA. J Phycol 40: 1028-1031

Miller AW, Ruiz GM, Minton MS, Ambrose RF (2007) Differentiating successful and failed molluscan invaders in estuarine ecosystems. Mar Ecol Prog Ser 332:41-51

Ng TH, Tan SK, Wong WH, Meier R, Chan SY, Tan HH, Yeo DC (2016) Molluscs for sale: assessment of freshwater gastropods and bivalves in the ornamental pet trade. PLOS ONE 11:e0161130

Panova M, Blakeslee AM, Miller AW, Mäkinen T, Ruiz GM, Johannesson K, André C (2011) Glacial history of the North Atlantic marine snail, Littorina saxatilis, inferred from distribution of mitochondrial DNA lineages. PLOS ONE 6:e17511

Patoka J, Bláha M, Kalous L, Vrabec V, Bu i M, Kouba A (2016) Potential pest transfer mediated by international ornamental plant trade. Sci Rep 6:25896

R Core Team (2018) R: a language and environment for statistical computing. R Foundation for Statistical Computing, Vienna

Romanuk TN, Zhou Y, Brose U, Berlow EL, Williams RJ, Martinez ND (2009) Predicting invasion success in complex ecological networks. Philos Trans R Soc B 364:1743-1754

Ruiz GM, Fofonoff PW, Steves BP, Carlton JT (2015) Invasion history and vector dynamics in coastal marine ecosystems: a North American perspective. Aquat Ecosyst Health Manage 18:299-311

Sá E, Costa PF, da Fonseca LC, Alves AS and others (2017) Trade of live bait in Portugal and risks of introduction of non-indigenous species associated to importation. Ocean Coast Manage 146:121-128

Schröder A, Nilsson KA, Persson L, Van Kooten T, Reichstein B (2009) Invasion success depends on invader body size in a size-structured mixed predation-competition community. J Anim Ecol 78:1152-1162

Seebens H, Schwartz N, Schupp PJ, Blasius B (2016) Predicting the spread of marine species introduced by global shipping. Proc Natl Acad Sci USA 113:5646-5651

Simard N, Plourde S, Gilbert M, Gollasch S (2011) Net efficacy of open ocean ballast water exchange on plankton communities. J Plankton Res 33:1378-1395

Editorial responsibility: Rochelle D. Seitz, Gloucester Point, Virginia, USA
Simkanin C, Davidson I, Falkner M, Sytsma M, Ruiz G (2009) Intra-coastal ballast water flux and the potential for secondary spread of non-native species on the US West Coast. Mar Pollut Bull 58:366-374

Smith E, van Belle G (1984) Nonparametric estimation of species richness. Biometrics 40:119-129

* Stachowicz JJ, Byrnes JE (2006) Species diversity, invasion success, and ecosystem functioning: disentangling the influence of resource competition, facilitation, and extrinsic factors. Mar Ecol Prog Ser 311:251-262

Stachowicz JJ, Tilman D (2005) Species invasions and the relationship between community saturation, diversity and ecosystem functioning. In: Sax DF, Stachowicz JJ, Gaines SD (eds) Species invasions: insights into ecology, evolution, and biogeography. Sinauer Associates, Sunderland, MA, p 41-64

* Tyrrell MC, Dionne M, Eberhardt SA (2012) Salt marsh fucoid algae: overlooked ecosystem engineers of north temperate salt marshes. Estuaries Coasts 35:754-762

* Valdivia N, Segovia Rivera V, Fica E, Bonta CC, Aguilera MA, Broitman BR (2017) Context-dependent functional dispersion across similar ranges of trait space covered by intertidal rocky shore communities. Ecol Evol 7:1882-1891

*Verling E, Ruiz GM, Smith LD, Galil B, Miller AW, Murphy KR (2005) Supply-side invasion ecology: characterizing propagule pressure in coastal ecosystems. Proc R Soc B 272:1249-1256

*Villéger S, Mason NWH, Mouillot D (2008) New multidimensional functional diversity indices for a multifaceted framework in functional ecology. Ecology 89:2290-2301

*Von Holle B, Simberloff D (2005) Ecological resistance to biological invasion overwhelmed by propagule pressure. Ecology 86:3212-3218

Wahl M (2009) Habitat characteristics and typical functional groups. In: Wahl M (ed) Marine hard bottom communities. Ecological Studies No. 206. Springer-Verlag, Heidelberg, p 7-17

Walther BA, Morand S (1998) Comparative performance of species richness estimation methods. Parasitology 116: 395-405

*Wonham MJ, Walton WC, Ruiz GM, Frese AM, Galil BS (2001) Going to the source: role of the invasion pathway in determining potential invaders. Mar Ecol Prog Ser 215:1-12

Zenni RD, Nuñez MA (2013) The elephant in the room: the role of failed invasions in understanding invasion biology. Oikos 122:801-815

Submitted: October 14, 2019; Accepted: March 18, 2020

Proofs received from author(s): April 30, 2020 